\title{
Clinical Study on Prevention, Treatment and Health Management of 2019-nCoV Pneumonia in China
}

\author{
Jun Meng* \\ Kangyang Industrial College, Sichuan University of Arts and Sciences, China
}

\begin{abstract}
The population of our country has exceeded 1.4 billion, and the epidemic situation of NCP is very serious now, so the clinical research on prevention, treatment and health management of NCP is not very perfect. In order to perfect the clinical medical research on the prevention and treatment of NCP and health management in China, the existing clinical medical research on the prevention and treatment of NCP and health management in China is still based on the medical view of "specialization and specialization", the negative effects of fragmentation of clinical knowledge and mechanization of clinical practice on the prevention and management of NCP have not been resolved. Based on the concept of integrative medicine, this paper puts forward a new concept of integrative medicine in clinical medical research on the prevention and treatment of 2019-nCoV pneumonia and health management. Emphasis should be placed on strengthening the integrated medical research and practice in the prevention, treatment and health management of NCP in China.
\end{abstract}

KEYWORDS: My country; 2019-nCoV pneumon; Prevention and health management; Clinical medicine

\section{INTRODUCTION}

In 2009, vice-president Fan daiming of the Chinese Academy of Engineering First put forward the brand-new medical concept and methodology of integrative medicine (HIM), which is also a brandnew health concept. From the perspective of holistic integration, he integrates the most advanced medical knowledge and the most modern medical experience in the field of human science, and in accordance with medicine, biology, chemistry, management, society, Physics, psychology, economy, physiology, environment, law, humanities and other sciences to continue to innovate, and eventually form a more conducive to human health of the advanced medical model. It is a scientific innovation, full integration of integrated medical theory. It is the ultimate goal of the innovative development of human medicine, a brand-new medical system, and an important guarantee for our country to actively promote the supply-side reform of the national health care and improve the national health care level. In order to do a better job in the reform of China's health care system, this paper, based on the concept of
Integrative Medicine, explores the establishment and improvement of China's new health care system.

The main problems in the clinical medical research on the prevention and management of NCP in China the main features of the clinical medical research on the prevention and management of NCP in China are as follows: These microscopic results cannot reflect the overall characteristics of the human body, the human body specimens to be transferred outside the body to explore, so that in vitro discovery cannot fully explain the situation inside the body; Conclusions drawn from the human anatomy sometimes do not really describe the functional characteristics of the human body and so on [1]. These clinical studies are of little significance to the clinical work of the prevention, treatment and health management of NCP. Advances in clinical research in the prevention, treatment and management of NCP should serve human health rather than focusing on publication, citation, and number of influencing factors. However, many clinical research papers on the prevention,
Quick Response Code:

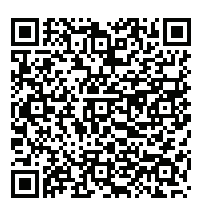

Address for correspondence: Jun Meng, Kangyang Industrial College, Sichuan University of Arts and Sciences, China

Received: April 08, $2021 \quad$ Published: May 05, 2021

How to cite this article: Jun M. Clinical Study on Prevention, Treatment and Health Management of 2019-nCoV Pneumonia in China. 2021- 3(3) OAJBS.ID.000275. DOI: 10.38125/OAJBS.000275 
treatment and management of NCP do not really contribute to human health. Main causes of existing problems in clinical medical research on the prevention, treatment and health management of new crown pneumonia in China [2] Has promoted the Infectious Disease Science Progress, has obtained the great achievement, has made the remarkable contribution for the human health. But its shortcomings and limitations are also becoming apparent. Bacon, Robert Koch, and Descartes contributed the most to the development of these methods, but they have their limitations.

Bacon's Thought: Science is omnipotent, and the omnipotent Materialist Dialectics tells us that every method and technology have its limitations. Science is a very good methodology, very useful, made a great contribution to mankind, but it is not omnipotent, and is not omnipotent. Bacon said, knowledge is power. Academician Fan daiming holds that knowledge is not yet a direct force, and there is still much work to be done in the middle. Only useful knowledge has power, and only knowledge has power through organic integration. Since the beginning of the 20th century, the number of infectious disease research papers has doubled or even increased dozens of times every 10 years. If these research papers are not integrated, it is difficult to have real application value, and some of them even lead medicine to the wrong way. Some medical experts were eager to apply these research papers to clinical work and put forward the concept of translational medicine in infectious diseases. They worked for more than ten years in the United States, but the results were not good and there was not much progress, because these scientific data are difficult to apply to the clinical work of infectious diseases department.

Robert Koch's three principles. Robert Koch was a famous pathologist who discovered the bacterium cholera. His three principles of etiology have been used until now, is the standard of medical peer review papers, not up to standard to supplement the experiment. If you want to prove that there is a causal relationship between $A$ and $B$, you must prove that a and $B$ must exist at the same time, that a must cause $B$, and that B must disappear if a is removed. These three principles are correct for infectious diseases caused by foreign causes, especially for the new coronavirus infection For example, the new coronavirus infection is caused by the new coronavirus, it is consistent with Robert Koch's three principles: First: The new coronavirus and new coronavirus infection at the same time; Second: New Coronavirus infection caused by new coronavirus infection; Third: The eradication of these new types of autoimmune coronavirus, new pneumonia infection will be cured. But Robert Koch's three principles do not apply to chronic diseases with multiple causes, such as alcohol consumption and chronic urticaria. The causal relationship is not established, according to Robert Koch's three principles.

First, both alcohol consumption and chronic urticaria exist, but many patients with chronic Urticaria do not drink; Second: Drinking can cause chronic Urticaria, but many people who drink alcohol rarely suffer from Chronic Urticaria; third: some patients with chronic urticaria recover after not drinking, but there are many patients with chronic urticaria do not drink the disease does not get better, and some even worse. Why does Robert Koch's rule of three not apply here? Because Chronic Urticaria can have multiple causes, not a single one. If we address one of the causes or if we remove the main cause, other causes will appear, and some will become the main cause. Robert Koch's three principles are based on logic, which says that two factors are the result of one direction. We often think of logic as cause and effect. Cause and effect have a lot of logic, but not just two factors, but a lot of factors; not just one direction, but a lot of directions, and some of them are networks. Each factor, each logic can support each other or cancel each other, is formed by many factors or the total amount of logic together as a whole result, so logic cannot be regarded as cause and effect.

Descartes's view Descartes is a methodological scientist of scientific research. He applied the scientific research method to the medical research, carried on the medical revolution, also made many rules for the medical research. Many of these rules are generally accepted to be correct, and there are three errors in medical research: First, the body-mind dualism, which separates the body from the mind and separates the body from the soul, which helps to move medicine away from theology and toward science; But are the data from human cells, tissues, and organs exactly the same as they actually are in the human body? It's definitely different. Can the body-mind dualism be right in its approach to understanding the laws of human life in terms of the many cell, tissue and organ studies that leave the human body? Second, Descartes's idea of "Cogito Ergo sum", in which he means that thinking about everything is only true if it is proven. The human mind is not omnipotent, the invisible is not necessarily absent, and this is not Idealism. Observers come to different conclusions when they look at the same thing from different angles. Descartes's theory has led to a lot of scientific research being repeated now, often making a mountain out of a molehill to prove something unimportant, which is missing the point. Third, Descartes believes that medicine, like science, breaks down the body into cells, tissues and organs, studies them from the beginning, and adds them up to an understanding of the body. However, the human cells, tissues, organs of a simple sum are not the whole of the human body, because the human body is a living life. The living human body is the whole human body, if there is no life, it is the human body. So, a living human body is divided into cells, tissues, organs, and no one lives. This is the contemporary over-segmentation of the medical profession, over-segmentation of the medical specialty, this specialist if the patient as a local focus to deal with the words, certainly not very good curative effect.

Chinese medicine-related research in Clinical Medical Research on the prevention and treatment of new coronary pneumonia and health management in China the COVID-19 of Chinese medicine and Western medicine in the prevention and treatment of new coronary pneumonia in China has preliminarily summarized some valuable experience and scientific research is still needed, continue to make a good summary and regular extraction. The Ministry of Science and Technology of the People's Republic of China has launched an urgent programme of scientific research on the prevention and control of the epidemic, including clinical research on traditional Chinese medicine and screening and evaluation of effective drugs. On February 3 this year, academician Zhang Boli was in charge of the National Science and Technology Emergency Project [3]: Clinical Research on the prevention and treatment of pneumonia caused by new coronavirus infection by integrated Chinese and Western medicine. The purpose of this study is first to serve clinical care, and second to do a good job of scientific analysis. The research will focus on the characteristics and evolution of COVID-19 syndrome, traditional Chinese and Western medicine treatment of common pneumonia (mild) clinical efficacy advantages, severe and severe pneumonia clinical value and Chinese medicine community prevention and control and rehabilitation. With the support of the Ministry of Science and Technology of the People's Republic of China and the Tianjin Science and technology bureau, screening and evaluation of listed proprietary Chinese medicines 
and their components have been carried out. The Tianjin University of Traditional Chinese Medicine has a state key laboratory for components of traditional Chinese medicine, which has a stockpile of nearly 60,000 components of traditional Chinese medicine and is currently conducting screening for active components with antiviral effects. Currently, active efforts are being made to screen and evaluate some Chinese patent medicines with anti-new coronavirus effects, such as Yinqiao Jiedu tablets, Lianhua qingwen granules, antiviral oral liquid, Jinhua Qinggan, Huoxiang Zhengqi, reduning, Qingkailing, Tanreqing, qingfeixiaoyan pills, antiviral granules, Jinggan capsules, Qingwenbaidusan and so on.

The Integrated Medical Research in clinical medical research on the prevention, treatment and health management of new coronary pneumonia in China has been studying the epidemic situation of new coronary pneumonia for more than two months. Medical and nursing personnel throughout the country have been going to successive "retrograde" epidemic areas, lighting up life with their lives, so that the new crown pneumonia of the epidemic prevention battle finally obvious effect! There are no bystanders, although there is panic to escape, but more people choose to bow into the game! As the Shanghai Jiao Tong University academician Chen Guoqiang wrote, "although pain is not fixed, we should also reflect on it. ". As medical professionals, we are duty-bound to consider such a serious question: Can we nip it in the bud? Can we prevent future disasters?". "This is the age of microorganisms, past, present, and future, until the end of the world... ", said world renowned biologist Stephen. John Jay. Gourd's conclusion about the nature of life on earth is that, yes, life on earth began with a single bacterium, and that our surroundings, including our human bodies, are full of a class one quadrillion virus, most of the time, these microbes live in peace with us humans, and when microbes cause disasters -- Epidemics of infectious diseases -- they often cross our borders, and it's time for us to take a moment to reflect on ourselves!

Let's turn our attention to the new coronavirus, which is the lowest form of life on a nanoscale, that causes us more pain than the bacteria, which are independent living organisms, that are fighting the human race, because of the invention of antibiotics, but the new coronaviruses are different. Two characteristics of the new coronaviruses make it difficult for us to overcome. First, the new coronaviruses cannot survive independently, it can only colonize living human cells, using human genetic material, and using that material inside the cells, to create a new coronavirus, and that's how it multiplies; when it infects humans, it integrates into the cells of our bodies, killing a new coronavirus means killing cells in the body, so for viruses that cause chronic diseases, such as hepatitis $B$, we can only use drugs that suppress the virus, such drugs are often taken for life, because as long as the drug is stopped, viruses will wait to reproduce, but such viruses have a low mutation rate with DNA (which is more stable than RNA), are easier to detect and identify, and vaccine control is a mature technology, the hepatitis $B$ virus, or retrovirus, has fallen dramatically since vaccination.

But the virus that causes acute infection is often RNA virus, it has the second characteristic "instability" of virus, this new coronavirus belongs to RNA virus, a detailed description of the new coronavirus can be found in the section on virological characteristics in the publication of the COVID-19, published by the National Health Commission, because of such characteristics, as the RNA virus replicates in the host, the mutation rate is also high, so the positive rate of the new coronavirus nucleic acid test remains to be evaluated, which makes the new coronavirus, like SARS, need to be re isolated and a vaccine developed, but time is of the essence, what happens to new coronaviruses when there is no chance of a vaccine waiting for them?

With the advent of the new coronaviruses, bats are once again in the public eye. This small, nocturnal creature, seemingly out of touch with our lives, is a mammal like us, and it's the only flying mammal on the planet, and because of their unique metabolic activity, and because of the bats 'gregarious nature, they fly with a lot of droplets coming out of their mouths, is very conducive to the existence and spread of the new coronavirus, and the most important point is the ancient nature of bats, bats are thought to have evolved at least 50 million years ago, so the history of bats carrying the virus is also very old, medical scientists have isolated nearly 4,000 viruses from nearly 200 species of bats, a natural reservoir of living viruses that evolved with the ancestors of mammals, it is well adapted to the conserved antigens common to some mammals, making the new coronavirus carried by bats easily transmissible between different mammals, including US, naturally, the SARS virus proved that bats were natural hosts (also known as storage hosts), while civets were proved to be intermediate hosts that eventually infected humans, a zoonosis that has happened more than once. The sequencing of the new coronavirus also confirmed a $96.2 \%$ similarity to a bat coronavirus, suggesting that the virus most likely originated in bats, on February 13 this year, Chinese researchers published a paper showing that they had found the intermediate host of the new coronavirus (COVID-19), Pangolin, which is the same key amino acid isolated from the smuggled pangolin in Guangzhou, THE VIRUS OF IN \& OUT PNEUMONIA has been clear, we have a tradition that Pangolin is a tonic, meat-based medicine, it is estimated that the seafood and game market in Wuhan will never be less Pangolin Shadow,... So, if we do not curb human desire, greed, if there is no fear of nature and life, if we do not change the habit of eating wild animals, such a disaster will be staged.

If this is a war without guns, "They Knew What They Wanted, 100 battles" should be the unchanging principle, when we focus all our efforts to analyze the new coronavirus thoroughly transparent, ready to open fire, it found that the available drugs and Technologies were so limited that, like SARS 17 years ago, there were no specific drugs, and the patient's own immunity was needed to overcome the new coronavirus, as the Internet celebrity put it, for the first time, "immunity" was the word of the people.

The immune system is often compared to the body's guard, immune cells like soldiers to invade the body's foreign microbes as the enemy to fight. Immune cells and organs in the "front line" to protect the body, the elimination of foreign small invaders, such as: bacteria, fungi, viruses, parasites, toxins, and so on, and resist the internal "renegade", such as tumors. Foreign Antigens are a target for the immune system, which sees them as not "our people, " a basic guarantee of human survival in this microbial-filled environment, natural Evolution has endowed the body's immune system with defensive function, stable (clean) function and monitoring function, forming the Great Wall Defense Line of human beings.

The Human Immune system usually reacts violently to a new coronavirus for the first time, and studies have shown that most SARS deaths are not directly caused by the virus itself, instead, he died from an overreaction of the immune system to an acute viral infection, such as Cytokine Storm, which Causes Acute Respiratory Distress Syndrome and multiple organ failure due to a strong immune response; The historical scene is strikingly similar today, with the clinical course of NCP again experiencing the same immune 
system overreaction as SARS, on Feb. 17, The Lancet published the case report of academician Wang Fusheng's team at the 301 Military Hospital, which performed the world's first pathological autopsy of a dead patient with new coronary pneumonia on Jan. 27 , the results showed a decrease in lymphocyte, but lymphocyte was also overactivated. In addition, the highly pro-inflammatory CCR4 + CCR6 + TH17 cells increase, which may explain the severe immune damage that patients exhibit in their lungs, suggesting that maintaining good immune homeostasis is particularly important in our fight against foreign invaders. "elevated levels of inflammatory cytokines are common in severe and critically ill patients, " according to a laboratory examination of the sixth edition of the COVID-19 on February 18, which also suggests that in patients with new coronary pneumonia, excessive Immune response is a major cause of deterioration or death in patients, and in this version of the treatment plan it is also proposed to treat critically ill patients with high inflammatory response, conditional can consider using plasma exchange, adsorption, perfusion, blood, plasma filtration and other in vitro blood purification technology, but these in vitro adjuvant treatment, only a few tertiary hospitals can complete,... A lot of patients just sit around and wait for treatment, and lose their window of opportunity... ? In the face of all this, we cannot help but think, the immune response to excessive inflammation, 17 years later, we are still so passive? Is there a way to balance our immune function in a way that only benefits the patient's body?

In fact, there is an answer to such a question, because human beings are the most perfect works of evolution in nature, and every organ of the body is working intelligently. However, in modern society, there are too many factors that interfere with the outside world. Slowly, in the background of these noises, the body slowly lost its original perfect, aging, disease appeared, and even in the face of new coronavirus invasion, lost the ability to fight back, then how to restore the best function of our body Integrative Medicine considers that human body is an organic and complex system, in order to maintain a stable state of balance, it will self-regulate. Any local changes are the result of system imbalance, the same system imbalance will be manifested in the local body; This is in line with the experience and theory of Chinese Medicine, which emphasizes the concept of individuation and wholeness and breaks down the traditional classification of diseases according to the principle of no harm to human body, to take nutrition, exercise, psychological, stress adjustment and other ways, so that our body to achieve their own balance.

In traditional Chinese Medicine (TCM), the balance of Yin and Yang is the basic principle. TCM emphasizes that "the vital energy in the body cannot be interfered with, and evil cannot be done. " In Western medicine, the vital energy can be understood as the aforementioned immunity, the interaction and cascade reaction of cytokines produced by oxidative stress of immune cells determine the dynamic process of disease course and are the dominant force to decide the disease condition and course.

The role of intestinal microecological therapy in the diagnosis and treatment of new coronary pneumonia has been emphasized in the third edition of the new coronary pneumonia diagnosis and treatment plan issued by the National Health Commission, "The requirement is to avoid blind or inappropriate use of antibiotics, especially in combination with broad-spectrum antibiotics, and to use intestinal microecology to maintain intestinal balance of nature and prevent secondary bacterial infections in the treatment of severe and critical cases. "Panels such as the Chinese Society for Nutrition and the Chinese Medical Association for enteral and parenteral nutrition have also recommended supplementation with probiotics to maintain intestinal microflora in the prevention and management of the balance of nature.

However, the "excessive use of antibiotics, Cesarean section and bottle feeding" that are prevalent in modern society have seriously affected the diversity of intestinal flora, it's a transient behavior during a new coronaviruses epidemic, and it's not recommended that we go back to normal in the future, and we shouldn't kill off the microbes, because the human immune system grows with the microbes. Mitochondria, the energy engine of life, are the specialized structures in cells that convert nutrients into energy. To cells, Mitochondria are like the engine of an airplane; except that red blood cells lack mitochondria, mitochondria can be found in almost all other cells, because the loss of mitochondrial function directly leads to cell damage and even death. Each cell of the body contains hundreds of Mitochondria, facing the virus, we often have to ask how to improve immunity, the most fundamental answer, improve mitochondrial function!

The Nature Paper demonstrates that different patterns of Mitochondrial metabolism support the effector function of differentiated helper $t$ cells (TH1) and specifically regulate the expression of genes involved in t cell activation, the quality of the Mitochondria directly affects the function of the immune system. We know that the number of Mitochondria in the body decreases with age, so protecting the quantity and function of Mitochondria is the most important factor to prevent immune aging. How do you protect mitochondrial function? Against free radicals. Along with the generation of mitochondrial energy, there are also free radicals that are harmful to the human body. Free radicals are produced in Mitochondria, which are prone to damage mitochondrial DNA. When the accumulation of ATP in mitochondrial tissues and DNA damage is reduced, cell function is impaired, this destructive process is similar to the oxygen that causes apples to turn Brown when they are cut open. Lack of cellular energy, along with energy expenditure, is associated with cell dysfunction, apoptosis, and inflammation, leading to tissue dysfunction, aging, and Degenerative disease. Clinically, many diseases, including some Neurodegeneration, are associated with mitochondrial damage, such as Alzheimer's disease, Parkinson's disease, dementia, autism, chronic fatigue syndrome, cardiovascular disease, diabetes, and Migraines.

The aforementioned "positive energy memory", implemented into specific body functions we call it "immunity", goes further and can be attributed to the Mitochondria' daily chain oxidative phosphorylation ATP production process, so the protection of Mitochondria is the body's constant supply of energy, so that our lives, "long live the Circle of Life! "!

People must ingest antioxidant food every day. The "Rainbow Diet" advocated now is the intake of fruits and vegetables rich in antioxidants. This is also in line with the theory of "five colors enter the five zang-organs" in Chinese medicine, many drugs are also common causes of mitochondrial damage and should be prevented in the prevention and treatment of NCP and health management in the future. Common Antioxidants include many plant compounds, such as Anthocyanins, lycopene, curcumin (the very same curry extract that Indians eat every day, curcumin can also inhibit the action of nfb molecules, so it's a natural anti-inflammatory nutrient), astaxanthin, Quercetin, green tea polyphenols, vitamin C, Vitamin E, now the internationally popular "Mediterranean Diet" is rich in antioxidants, Omega-3 unsaturated fat, anti-inflammatory 
Diet, so in order to improve immune homeostasis, avoid immune aging, omega-3 Essential fatty acid, coenzyme Q10, vitamin D and so on are all essential key factors. In February 12, the State Council "common war and epidemic" joint prevention and control press conference said: Nutrients for health, prevention, treatment and rehabilitation of diseases, especially for the prevention and treatment of new pneumonia and health management!

Whether the new coronavirus will be normalized or not, it is difficult to say at this time. We need to continue to strengthen the epidemiological investigation and basic research, after all, we have limited knowledge about it. However, the impact of the coronavirus on human society will never end. It has been relatively benign for most of its existence since its discovery in the 1960s, but several mutations in the past two decades have led to large-scale epidemics and heavy losses. Therefore, we need to continue to study the pathogenesis of coronavirus infection, research and development of broad-spectrum anti-coronavirus drugs, so as not to change. And now our country is doing the same thing. Compared with SARS, the new coronavirus is more "cunning", more "changeable", but also more "unpredictable. In the early stages, the disease doesn't seem to be very serious, but at a certain stage, it can go downhill rapidly. The new coronavirus is more transmissible than SARS, and the number of cases and deaths are much more than SARS. And the patient after cure can appear "repeatedly", have certain proportion of the patient after discharge again "Fu Yang". Rehabilitation of critically ill patients is also more complicated than SARS. But we still want to have confidence, after all, discharged from the hospital, "Fu Yang" is a minority of patients. Moreover, "Fu Yang" patients' treatment is also relatively easy, the basic treatment of a few days will be "turned negative. Rehabilitation will also be the focus of our research in the future. New Crown Pneumonia Prevention and Health Management Hwaseo Hospital clinical experience 1.5 hours can be simultaneously tested for six viruses, high cure rate, zero medical infection, sichuan University Hwaseo hospital brings to its director Hwaseo experience in the prevention, treatment and health management of NCP: Zero Hospital infection, high cure rate, innovative virus detection... in the battle against NCP, hwaseo hospital has perfected the Hwaseo culture of "family, country, common people". How to make a good deployment of epidemic prevention? How to prevent and control nosocomial infection? How to improve the accuracy of early diagnosis of new coronary pneumonia and the success rate of treatment in critically ill patients? Li Weimin, head of the expert group on medical treatment of severe cases of new coronary pneumonia in Sichuan Province, has brought us the "Sichuan University Hwaseo experience".

Prompt implementation of the prevention and control of new coronavirus infection in hospitals to ensure zero infection among healthcare workers, "SARS, influenza a (H1N1), avian influenza... Previous outbreak of respiratory infectious diseases prevention and control experience tells us that good hospital and medical infection prevention and control is crucial, "said Li Weimin, president of the hospital, the prevention and control of new coronavirus infection in hospitals is an important part of the prevention and control of new coronavirus infection, whether from the aspect of protecting medical staff, it is also important to protect non-ncp patients in other hospitals and to reduce the risk of aggregated transmission. Therefore, in the early stage of new crown pneumonia, Hwaseo hospital launched a series of prevention and control measures, and summarized the experience as "three separate", "three levels of preexamination" and "three norms".
"Three separations" include separation of in-patients, isolation of wards with separate spaces for newly diagnosed or suspected pneumonia patients, separation of general emergency patients from fever outpatients; General outpatients are separated from fever outpatients. Three-level pre-examination and triage can effectively avoid the cross-infection of the crowd in the hospital. The first-level pre-screening and triage service is located at the entrance of the hospital's out-patient and emergency departments. It is mainly used for temperature monitoring and screening of patients entering the out-patient Department of the hospital's emergency departments, as well as simple epidemiological and respiratory system symptom inquiries and surveys, those with fever (body temperature over $37.3^{\circ} \mathrm{C}$ ) or a history of epidemiology are immediately escorted to the fever clinic of the hospital for treatment.

The second-level pre-examination triage is located in the ward of the hospital, and the body temperature is taken again by the nurses' stations at all levels, the key departments of the hospital, such as the Department of aspiration medicine, the Department of Infection, the Department of Integrated Chinese and Western medicine, will also ask about their epidemiological history; the third-level pre-examination and triage service will be set up in the hospital consultation room, and the doctors in the outpatient department will ask again when receiving patients, for those with fever or a history of epidemiology, inform the nurse of the clinic immediately and escort them to the fever clinic by the nurse of the clinic. The "three norms" first regulate personal protection in hospitals. Medical workers in hospitals should do a good job of selfprotection, including correctly wearing masks and standardizing handwashing and disinfection.

For staff in high-risk positions in hospitals, they should wear protective clothing, wear Goggles; every department and Department in the hospital will conduct rigorous epidemiological inquiries and investigations. Next is the standard patient sample collection, in the collection process is particularly easy to cause the medical worker's infection, how faster, more accurate, less pollution to carry on the related examination, has a series of standard operating procedures. The third is the standardized transfer of new pneumonia patients, which needs to be carried out in strict accordance with the hospital's transfer work plan.

An hour and a half to test for six viruses at the same time, the Sichuan University Hwaseo hospital innovation to help early diagnosis, early treatment, February 22 this year, six respiratory virus nucleic acid test kits, including the new coronavirus, developed by Hwaseo Hospital in collaboration with Tsinghua University, have been approved by the State Food and Drug Administration for the second batch of new coronavirus emergency medical devices, for many patients and front-line medical staff to provide rapid, accurate and effective diagnosis. "As academician Zhong Nanshan said, this is the peak season of influenza. It is the easiest time to mix the flu with the COVID-19.

How to distinguish it quickly, make a good differential diagnosis and target treatment is very important, " Li said, the approved kit uses a thermostatic amplification chip method, which simply takes samples of the patient's nasal and pharyngeal swabs and other secretions, six common respiratory viruses, including NCPV, can be detected in one and a half hours at a time. The Kit has been emergency support to major hospitals throughout the country, especially in Wuhan and other areas of severe new coronavirus infection [3-6]. 


\section{REFERENCES}

1. Fan DM (2017) Integrative medicine: Theory and practice [m]. World Book Publishing Company, China.

2. Lanjuan L, Fan DM (2019) Integrative medicine: Theory and practice $5[\mathrm{~m}]$. World Book Publishing Company, China.

3. Zhang B, Fan DM (2017) Integrative Medicine: Theory and practice [m] World Book Publishing Company, China.
4. Huang L, Fan DM (2017) Integrative medicine: Theory and practice $2[\mathrm{~m}]$. World Book Publishing Company, China.

5. Wu Y and Fan DM (2019) Integrative medicine: Theory and practice $6[\mathrm{~m}]$. World Book Publishing Company, China.

6. Tsang K (2011) Introduction to functional medicine [m]. People's Medical Publishing House, Beijing, China. 